\title{
STIS spectroscopy of newborn massive stars in SMC N81^
}

\author{
M. Heydari-Malayeri ${ }^{1}$, M. R. Rosa ${ }^{2, \star \star}$, D. Schaerer ${ }^{3}$, F. Martins ${ }^{3}$, and V. Charmandaris ${ }^{4}$ \\ 1 DEMIRM, Observatoire de Paris, 61 avenue de l'Observatoire, 75014 Paris, France \\ 2 Space Telescope European Coordinating Facility, European Southern Observatory, \\ Karl-Schwarzschild-Strasse-2, 85748 Garching bei München, Germany \\ 3 Laboratoire d'Astrophysique, Observatoire Midi-Pyrénées, 14 avenue É. Belin, 31400 Toulouse, France \\ ${ }^{4}$ Cornell University, Astronomy Department, 106 Space Sciences Bldg., Ithaca, NY 14853, USA
}

Received 14 August 2001 / Accepted 25 October 2001

\begin{abstract}
Using Hubble Space Telescope observations with STIS, we study the main exciting stars of N81, a high excitation compact H II region in the Small Magellanic Cloud (SMC). These far UV observations are the first spectroscopic measurements of stars in such a region and reveal features characteristic of an O6-O8 stellar type. The astonishing weakness of their wind profiles and their sub-luminosity (up to $\sim 2$ mag fainter in $M_{V}$ than the corresponding dwarfs) make these stars a unique stellar population in the Magellanic Clouds. Our analysis suggests that they are probably in the Hertzsprung-Russell diagram locus of a particularly young class of massive stars, the so-called $\mathrm{Vz}$ luminosity class, as they are arriving at the zero age main sequence.
\end{abstract}

Key words. stars: early-type - ISM: dust, extinction - ISM: H II regions - ISM: individual objects: N81 - galaxies: magellanic clouds

\section{Introduction}

Understanding the formation of massive stars, which is still a largely unsolved problem, requires studying them at the earliest phases where they can be reached through the enshrouding material at different wavelengths. While high resolution radio continuum observations allow the investigation of ultracompact $\mathrm{H}$ II regions formed around newborn massive stars (Churchwell 1990), high angular resolution observations in the ultraviolet, visible, and infrared are also necessary to access accurate physical parameters of these stars in order to identify their evolutionary states (Walborn \& Fitzpatrick 1990; Walborn et al. 1995b; Hanson et al. 1996). In particular, UV observations are of prime importance since massive stars emit the bulk of their energy in this wavelength range. In practice, though, observing newborn massive stars is not straightforward for

Send offprint requests to: M. Heydari-Malayeri, e-mail: heydari@obspm.fr

* Based on observations with the NASA/ESA Hubble Space Telescope obtained at the Space Telescope Science Institute, which is operated by the Association of Universities for Research in Astronomy, Inc., under NASA contract NAS 5-26555.

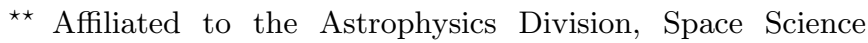
Department of the European Space Agency. several reasons. Mainly, they are very rare, and the relatively small evolutionary timescales involved make it difficult to catch them just at this very point in their evolution, that is when they become observable in the UV and visible (Yorke \& Krügel 1977; Shu et al. 1987; Palla \& Stahler 1990; Beech \& Mitalas 1994; Bernasconi \& Maeder 1996).

We have amply argued that the compact HiI regions known as HEBs (High Excitation Blobs) provide the best opportunities for a direct access to massive stars at very early stages of their evolution (Heydari-Malayeri et al. 2001a and references therein). The members of this distinct and very rare class of ionized nebulae in the Magellanic Clouds are small and compact $\left(\sim 5^{\prime \prime}\right.$ to $10^{\prime \prime}$ in diameter corresponding to $\sim 1.5-3.0 \mathrm{pc}$ ), in contrast to the typical H II regions in those galaxies, which are extended structures (sizes of several arcmin corresponding to more than $50 \mathrm{pc}$, powered by a large number of exciting stars). In general, HEBs are also heavily affected by local dust, as one would expect from their very young age (HeydariMalayeri et al. 2001a and references therein, see also Israel \& Koornneef 1991). Also their study is pertinent to understanding the process of massive star formation, especially in the context of the Magellanic Clouds.

Our recent high-resolution imaging with the Hubble Space Telescope (GO 6563, GO 8246) using the Wide Field Planetary Camera (WFPC2) has for the first time resolved 
several HEBs which had appeared featureless to groundbased telescopes: SMC N81, N88A, LMC 159-5 (the Papillon nebula), N83B, and N11A (Heydari-Malayeri et al. 1999a, 1999b, 1999c, 2001a, 2001b). The HST observations uncover the stellar content hidden thus far, as well as the nebular features of these compact nebulae and display a turbulent environment typical of newborn massive star formation sites: outstanding emission ridges created by shocks and cavities sculpted in the ionized gas by the powerful winds of massive stars, prominent dust structures protruding from hot gas. The observations also bring to light even more compact H II blobs, immersed in the HEBs, harboring newborn, hot stars.

The present paper is devoted to N81, also known as DEM 138 (Henize 1956; Davies et al. 1976), a nebula only $\sim 10^{\prime \prime}$ across and located in the Shapley Wing at $\sim 1.2(\sim 1.2 \mathrm{kpc})$ from the main body of the SMC. A first detailed study of this compact $\mathrm{H}$ II region, carried out by Heydari-Malayeri et al. (1988), revealed its nature and some of its physical characteristics: gas density and temperature, chemical composition, mass, age, etc. Subsequently, near infrared observations showed the presence of $\mathrm{H}_{2}$ emission towards N81 (Israel \& Koornneef 1988), while ${ }^{12} \mathrm{CO}(1-0)$ emission at two points towards this HII region was also detected (Israel et al. 1993). However, due to the lack of sufficient spatial resolution, it was not possible to view and study the exciting star(s) hidden inside the ionized gas. Therefore, the rather important question, which is often raised by star formation theories, of whether N81 was powered by a single massive star or a cluster of them, remained unanswered. This is, however, a critical question for theories of star formation.

High spatial resolution imaging with HST allowed us to resolve N81 and revealed the presence of a tight cluster of newborn massive stars embedded in this compact nebula (Heydari-Malayeri et al. 1999, hereafter Paper I). Six of the stars are grouped in the core region of $\sim 2^{\prime \prime}$ diameter, with a pair of the main exciting stars in the very center separated by only $0^{\prime \prime} .27$ or 0.08 pc. The images also displayed conspicuous marks of strong stellar winds, shocks, and ionization fronts characterising turbulent massive star forming regions. Moreover they revealed prominent dust lanes dividing the nebula into three lobes. One of the lanes, running over $15^{\prime \prime}(4.5 \mathrm{pc})$, ends in a magnificent curved plume. A remarkable absorption "hole" or dark globule of radius $\sim 0^{\prime \prime} .25(\sim 0.07 \mathrm{pc})$ is situated towards the center of the $\mathrm{H}$ II region, where the extinction reaches higher values $\left(A_{V}=1.3 \mathrm{mag}\right)$. These absorption features are probably parts of the molecular cloud which have given birth to the massive stars.

From the Strömgren uvby imaging with WFPC2 we carried out the photometry of some 50 stars towards N81. This allowed us, using color-magnitude diagrams, to select the main exciting stars of the region. This paper is devoted to the spectroscopy of these stars. We derive spectral classification for these very young massive stars and study their nature.

\section{Observations and reduction}

The General Observer Program No. 8246 devoted to observations of N81 was performed with Space Telescope Imaging Spectrograph, STIS (Woodgate et al. 1998) on board HST on 28 and 31 October 1999. The spectra were obtained with the far-UV Multi-Anode Microchannel Array (MAMA) detector in the G140L mode covering the wavelength range $1120-1715 \AA$. All the observations were made through the $52^{\prime \prime} \times 0^{\prime \prime} \cdot 2$ entrance slit. The effective resolution was $0.6 \AA$ per pixel of $25 \mu \mathrm{m}$, corresponding to a dispersion of $24 \AA \mathrm{mm}^{-1}$, or a resolution of $1.2 \AA$ $(F W H M)$. The exposure times were set according to the apparent magnitudes of the stars in order to equalize the signal-to-noise ratios $(S / N)$ of the spectrograms. Total exposure times varied from $1229 \mathrm{~s}$ (stars \#1 and \#2) to 3169 s (stars \#3, \#4, and \#8). Three relatively faint stars (\#5, \#7, \#10), not initially scheduled for observations, happened to lie on the slit when observing their adjacent stars (\#3 and \#11). The $S / N$ ratio is particularly weak for these stars, yet we present the spectrogram of star \#5 which shows some interesting features. STIS was also used to obtain the spectra of the N81 stars in the visible domain. The grating G430L covered the range of 2900 to $5700 \AA$ with a resolution of of $2.73 \AA$ per pixel. The CCD pixels of $21 \mu \mathrm{m}$ yielded a dispersion of $130 \AA \mathrm{mm}^{-1}$. The exposure times ranged from $24 \mathrm{~s}$ (star \#1) to $750 \mathrm{~s}$ (star \#8).

The calibrated output products from the standard pipeline use a default extraction aperture of 22 pixels ( $0^{\prime \prime} 53$ on the sky). We carefully reprocessed the 2D images using the most recent calibration reference files applicable to the observations and extracted the spectra using slits of both 6 and 2 pixels. We verified the centering of the stars on the slits, and tested for the effects of different sky background extraction on our spectra. The 6 pixel slit yielded spectra which are very similar to those produced by the standard pipeline with an insignificant loss in $S / N$. Even the 2 pixel slit did not indicate any extraction effect due to its size other than the expected loss in $S / N$. Comparing the resulting line profiles as a function of the aperture width, we found that other than an increase in noise when going to the smaller slits, and slightly modifying the slope of the spectra (because of a slight tilt of the spectral images), the line profiles do not change by any significant amount. The spectra displayed in Figs. 1 and 2 are based on 6 pixel extraction apertures.

\section{Results}

The N81 stars observed with STIS are listed in Table 1, and their physical location can be seen in Fig. 2 of Paper I. The table also presents the corresponding photometry of the stars (Paper I). The color excesses $E(B-V)$ were derived from $E(b-y)$ using the intrinsic color $(b-y)_{0}=$ -0.15 mag for hot stars (Relyea \& Kurucz 1978) and assuming that our observed colors represent the standard Strömgren system. The relation $E(B-V)=1.49 E(b-y)$ 
Table 1. SMC N81 stars observed with STIS.

\begin{tabular}{ccccccc}
\hline Star number & $\begin{array}{c}\alpha \\
(\mathrm{J} 2000)\end{array}$ & $\begin{array}{c}\delta \\
(\mathrm{J} 2000)\end{array}$ & $\begin{array}{c}y \\
(\mathrm{~F} 547 \mathrm{M}) \\
(\mathrm{mag})\end{array}$ & $\begin{array}{c}b-y \\
(\mathrm{mag})\end{array}$ & $\begin{array}{c}A_{V} \\
(\mathrm{mag})\end{array}$ & $\begin{array}{c}M_{V} \\
(\mathrm{mag})\end{array}$ \\
\hline 1 & $01: 09: 13.1$ & $-73: 11: 38.3$ & 14.38 & -0.10 & 0.22 & -4.84 \\
2 & $01: 09: 13.0$ & $-73: 11: 38.0$ & 14.87 & -0.11 & 0.19 & -4.32 \\
3 & $01: 09: 13.4$ & $-73: 11: 38.4$ & 16.10 & -0.08 & 0.31 & -3.21 \\
4 & $01: 09: 12.8$ & $-73: 11: 38.3$ & 17.41 & +0.07 & 1.02 & -2.61 \\
5 & $01: 09: 13.3$ & $-73: 11: 37.6$ & 18.29 & -0.05 & 0.46 & -1.17 \\
8 & $01: 09: 12.8$ & $-73: 11: 40.2$ & 17.84 & +0.15 & 1.40 & -2.56 \\
11 & $01: 09: 13.7$ & $-73: 11: 33.3$ & 15.74 & -0.10 & 0.22 & -3.48 \\
13 & $01: 09: 16.1$ & $-73: 11: 29.1$ & 16.65 & -0.08 & 0.31 & -2.66 \\
\hline
\end{tabular}

(Kaltcheva \& Georgiev 1992) was then used to transform into the Johnson system, which finally yielded the extinctions $A_{V}=3.1 E(B-V)$. The estimated absolute magnitudes are based on a distance modulus $M-m=$ $19.0 \mathrm{mag}$ (corresponding to a distance of $63.2 \mathrm{kpc}$, e.g. Di Benedetto 1997 and references therein) and assuming that the Strömgren $y$ filter is equal to the Johnson $V$.

The final reduced spectrograms are presented in Figs. 1 and 2, where the former figure includes the four brightest stars of the sample, whereas Fig. 2 displays the fainter ones. The main stellar features (C III $\lambda 1176, \mathrm{NV} \lambda 1239$, 1243, Ov $\lambda 1371$, Si Iv $\lambda \lambda 1394,1403$, C IV $\lambda$ 1548, 1551, and He II $\lambda 1640$ ) are distinguished with tick marks. The labels appearing below the features indicate cases where contamination with an interstellar component is possible. The identification of S V $\lambda 1502 \AA$ is based on the work of Werner \& Rauch (2001).

An outstanding aspect of these spectra is the extreme weakness of the UV wind profiles. Weak stellar wind features in SMC O stars have already been found by several workers (Hatchings 1982; Garmany \& Conti 1985; Walborn et al. 1995b), who ascribed them to the metal deficiency of the SMC leading to a reduced radiation pressure responsible for driving the winds of early-type stars. More recent observations have further confirmed this result (Smith Neubig \& Bruhweiler 1997; Walborn et al. 2000). However, the wind features observed in the stars of N81 are even weaker. If we consider the usually stronger wind lines seen in O stars (such as the N v $\lambda 1239,1243$ and C IV $\lambda 1548,1551$ in dwarfs, or the Si IV $\lambda \lambda 1394,1403$ in giants/supergiants), with the exception perhaps of star \#5, none shows the emission part of a P-Cygni profile and the absorption is extremely weak, particularly for $\mathrm{N} v \lambda \lambda 1239$, 1243. For stars which we classify as O types (see below), such a behavior is observed for the first time.

\subsection{Spectral classification}

Traditionally the spectral classification schemes of stars are based on their optical spectra. However, recent studies comparing spectral features in the optical and UV have resulted in a global, coherent picture of classification criteria (Walborn et al. 1985, 1995a; Smith Neubig \& Bruhweiler 1997, 1999). In particular,
Smith Neubig \& Bruhweiler (1997) have proposed a UV classification system for $\mathrm{O}$ and $\mathrm{B}$ stars of the SMC which is defined by a set of standard, low resolution spectra observed with the International Ultraviolet Explorer (IUE). This UV scheme, which was used by the authors to derive classifications for $133 \mathrm{O}$ and B stars of the SMC, while independent of the MK system, shows general agreement with those deduced from visual data.

The low $S / N$ ratio of our STIS optical spectra and in particular their contamination with strong nebular emission lines limit their practical use for spectral classification. This can be understood since N81 is a very bright compact $\mathrm{H}$ II region with strong nebular emission lines in the visible part of the spectrum (Paper I). Although nebular emission lines are present also in the UV part, they are much less troublesome. Therefore, we will use the method put forward by Smith Neubig \& Bruhweiler (1997). However, given some morphological differences of the N81 UV spectra with previously well studied stars, the limitations of the optical part of the spectrum mentioned earlier, and the constraints available from the UV classification scheme (Smith Neubig \& Bruhweiler 1997), it is clear that there is no unique solution to the spectral classification of our targets.

The presence of HeII $\lambda 1640$ and $\mathrm{OV} \lambda 1371$ in all spectra, except perhaps in stars \#13 and \#8 which have lower $S / N$ ratios, is the first and strongest evidence that we are observing $O$ type stars. These spectra though display characteristics suggesting that the stars also belong to the dwarf luminosity class since the features Si IV $\lambda \lambda 1394,1403$ as well as N v $\lambda \lambda 1239,1243$ and C IV $\lambda \lambda$ 1548, 1551 are weak (even weaker than usual). Star \#5 may be a different case as explained below. These features are known to increase with luminosity, ranging from weak P-Cyg profiles on the main sequence to very pronounced P-Cyg profiles in the supergiants (Walborn et al. 1995a; Smith Neubig \& Bruhweiler 1997). A dwarf luminosity class is also supported by the optical spectra which show no He II $\lambda 4686$ and N III $\lambda 4640$ emission. Although the morphology of the N81 spectra differs qualitatively from that of the known $\mathrm{O}$ types, we may classify them as "zero age main sequence dwarf" Vz, based on the weakness of the wind lines (Walborn \& Parker 1992; Walborn \& Blades 1997; Walborn et al. 2000). Note that 


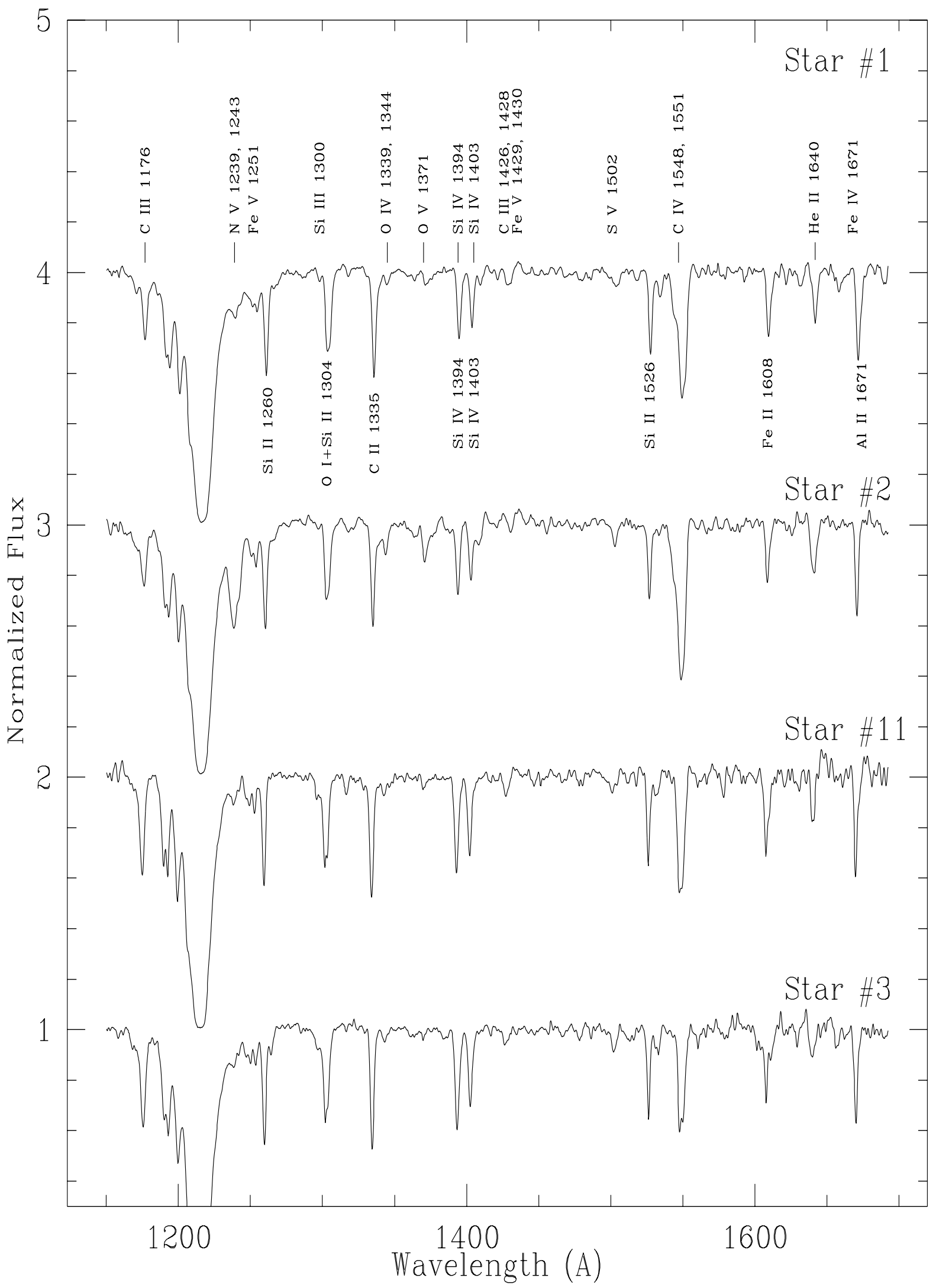

Fig. 1. Rectified HST/STIS ultraviolet spectrograms of the four brightest stars in SMC N81. The prominent absorption feature at $\lambda 1210 \AA$ is due to the $\operatorname{Ly} \alpha$. The wind profiles are indicated with tick marks and the features possibly contaminated by an interstellar component are labelled below the lines. 
M. Heydari-Malayeri et al.: STIS spectroscopy of newborn massive stars in SMC N81

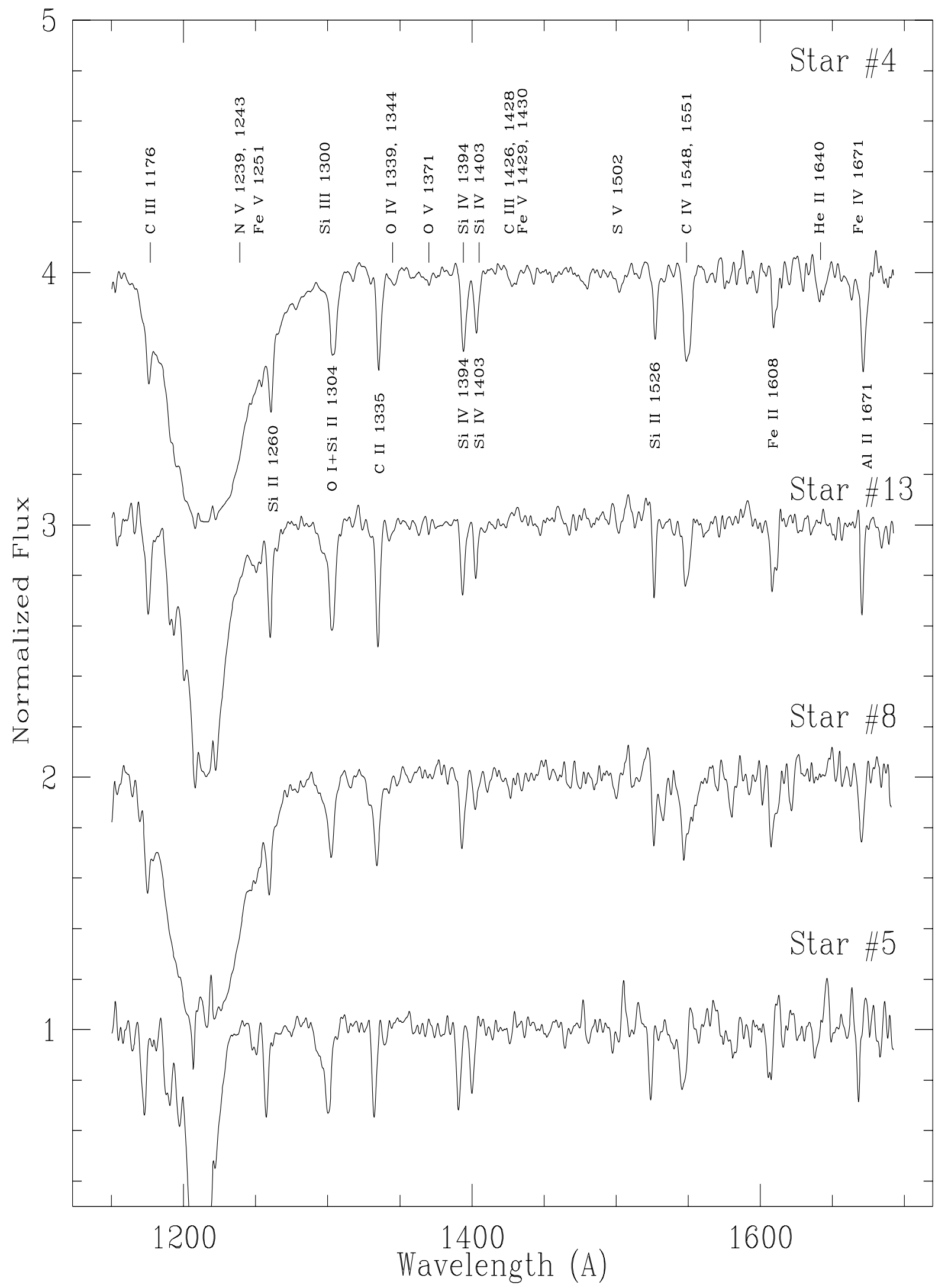

Fig. 2. Spectrograms for the remaining four less bright stars of N81. The notation is the same as in Fig. 1.

the original definition of the $\mathrm{Vz}$ class is He II $\lambda 4686 \mathrm{ab}-$ and therefore the association of these stars with that class sorption much stronger than He II $\lambda 4541$ or HeI $\lambda 4471$, is indirect. 
No clear distinction between the various subtypes can be made based on the available spectral features, but it is very likely that all stars are of a late O type ( O $6-\mathrm{O} 8)$. This is supported by the following three facts. First, the presence of the $\mathrm{OV} \lambda 1371$ feature indicates a spectral type earlier than $\lesssim$ O8 (Smith Neubig \& Bruhweiler 1997). Second, the weakness of the Si III $\lambda 1300$ feature, which appears in the wing of O I + Si II $\lambda$ 1304, excludes much later types. Finally, the N v $\lambda \lambda 1239,1243$ feature is weaker than $\mathrm{C}$ IV $\lambda \lambda 1548,1551$, which is only seen in $\mathrm{O}$ types $\geq \mathrm{O} 6-\mathrm{O} 7$ or alternatively in OC stars (Walborn \& Panek 1985).

Star \#5 shows some puzzling emission features in its spectrum $(\lambda \lambda 1480,1508,1616$, and $1640 \AA)$, at least one of which, He II $\lambda 1640$, is apparently part of a P-Cyg profile. It should be stressed though that the $S / N$ ratio is not high enough to be absolutely certain about their presence. If these were indeed wind induced features, their presence would confirm the fact that the $\operatorname{CIV} \lambda \lambda 1548,1551$ profile has a particularly marked emission component compared to the other stars of the sample. Since both of the He II $\lambda 1604$ and Sv $\lambda 1502$ lines show P-Cyg profiles in Of and Wolf-Rayet spectra (Walborn et al. 1985; Willis et al. 1986), star \#5 appears to be an Of or WR candidate in $\mathrm{N} 81$.

\subsection{Stellar parameters and wind properties}

To constrain the stellar wind properties we have examined the line profiles of the strongest UV lines. The best indications for velocity shifts come from the C IV $\lambda 1548$, 1551 feature. The profiles of stars \#1 and \#2 show blueshifted absorption reaching up to velocities of $\sim 1700$ and $2000 \mathrm{~km} \mathrm{~s}^{-1}$ respectively. These values are compatible with terminal velocity measurements in other SMC O stars of similar spectral type (Walborn et al. 1995b; Prinja \& Crowther 1998). For star \#5 we derive a terminal velocity of $1000 \mathrm{~km} \mathrm{~s}^{-1}$, while blue-shifts with smaller velocities are seen in the remaining profiles. However, these profiles also show asymmetries which are more important on the red side. Further investigations will be necessary to understand the detailed behavior of the line profiles.

Using an effective temperature derived from the estimated spectral types (O6-O8), in particular the one from Vacca et al. (1996) for dwarfs, and the absolute magnitudes from Table 1, we place the N81 stars (filled circles) in an HR-like diagram (Fig. 3). For further comparison we also include the mean $M_{V}$ magnitudes for O3-B0 dwarfs based on a compilation by Vacca et al. (1996), as well as the mean $M_{V}$ relation of Walborn (1972) used by Walborn \& Blades (1997) for 30 Doradus stars. Figure 3 clearly shows that most of the stars in N81 are sub-luminous compared to the mean $M_{V}$ for dwarfs. This finding still holds even if the spectral types are shifted by 1-2 subtypes towards later types. Compared to the so-called Vz luminosity class, the sub-luminosity of the N81 stars is more pronounced. Our targets are up to $\sim 2$ mag fainter in $M_{V}$ than the mean relation for dwarfs! Although this is not

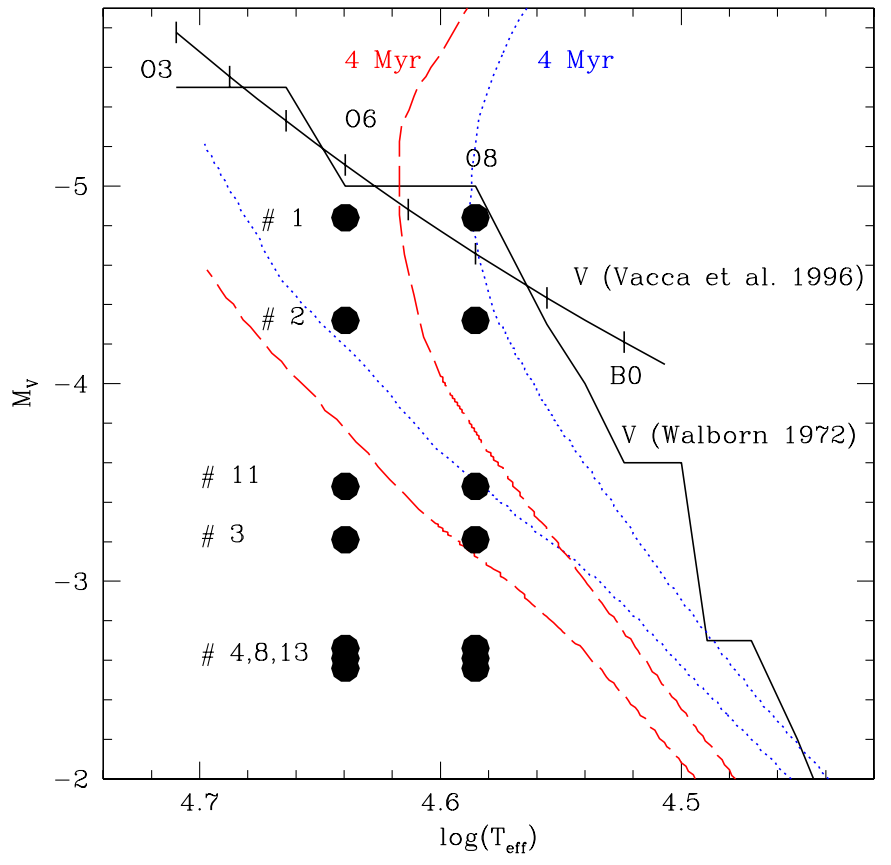

Fig. 3. The absolute $M_{V}$ magnitude versus effective temperature diagram of the N81 stars is compared to the zero age main sequence (ZAMS) and $4 \mathrm{Myr}$ isochrones at various metallicities. The $T_{\text {eff }}$ of the stars has been estimated using an $\mathrm{O} 6$ or $\mathrm{O} 8$ spectral type and the Vacca et al. (1996) scale. The ZAMS and $4 \mathrm{Myr}$ isochrones for a metallicity of $1 / 20 Z_{\odot}(Z=0.001)$ are indicated by long-dashed lines while the same pair for $1 / 5 Z_{\odot}$ $(Z=0.004)$ is plotted with dotted lines. The ZAMS curves are not plotted for $\log \left(T_{\text {eff }}\right) \geq 4.7$ due to lack of an appropriate conversion to $M_{V}$. Also shown, as solid lines, are the $M_{V}-T_{\text {eff }}$ calibration for dwarfs from Vacca et al. (1996) as well as the one from Walborn (1972). Note that four of the observed stars are situated in the HRD-like diagram in a locus suggesting that they are either on the ZAMS or that they have a young age. The lower luminosity stars $(\# 4,8,13)$ are possibly hotter than the ZAMS.

the defining characteristic of the Vz class, the above indications as well the weakness of the UV wind lines further attest to the possibility that these stars belong to the $\mathrm{Vz}$ class.

Two sets of ZAMS and isochrones of 4 Myr are presented in Fig. 3 using the Geneva stellar evolution tracks for metallicities bracketing approximately that of the SMC (Lejeune \& Schaerer 2001). One set has been computed for a metallicity $1 / 20$ Solar $(Z=0.001)$ and is marked with a long-dashed line, while the second has a metallicity of $1 / 5$ Solar $(Z=0.004)$ and is marked with a dotted line. The observed $M_{V}$ and spectral types are roughly compatible with positions close to the theoretical ZAMS or young ages. Given mostly the lack of an accurate spectral subtype determination we cannot firmly establish if the lower luminosity stars (\#8, \#13) are really hotter than the ZAMS. Atmospheric modeling is in progress to obtain more accurate stellar parameters of these unique young stars in the SMC (Martins et al., in preparation). Based on the $Z=0.004$ tracks, the ZAMS luminosities and 
masses corresponding to the observed $M_{V}$ are between $\log L / L_{\odot} \sim 4.2-5.5$ and $\sim 14-50 M_{\odot}$ respectively.

\section{Discussion}

The HST spectra of N81 presented here are the first ones ever obtained from a tight cluster of stars in a HEB. The reason is that these stars, embedded in a compact emission nebula, have not been reachable by ground-based telescopes. And even with recent developments in groundbased instruments, taking spectra of individual stars in the visible remains still practically infeasible. As a result, contrary to other massive stars in the SMC that have been observed from space in the UV (Walborn et al. 1995b, 2000; Smith Neubig \& Bruhweiler 1997), the N81 stars lack high quality spectra in the visible. Our low-resolution $H S T$ spectra in the visible, imposed by stringent time allocation constraints, were intended to be a first exploratory attempt.

Massive stars observed from the ground and also with $H S T$ are typically much brighter than the ones seen in N81 and comparably they are much less affected by nebular emission and dust. The decoupling from nebulae is presumably due to the evolutionary state of these stars; they have had enough time to entirely and/or effectively disrupt their $\mathrm{H}$ II regions and the associated dust. This means that those bright stars are older than the N81 members, as supported by their spectra. Among the 15 SMC stars studied by Walborn et al. (2000), 9 are clearly giants, 5 are peculiar and have already developed emission line features of N IV $\lambda 4058, \mathrm{~N}$ III $\lambda 4640$, or C III $\lambda 4650$ (two of them being on the main sequence), and the last one is a pure main sequence.

The fact that all the observed exciting stars of N81 display the $\mathrm{Vz}$ characteristics further supports the very young age of the cluster (Paper I). This observed concentration of $\mathrm{Vz}$ stars in a small region is intriguing since in the LMC region of 30 Doradus only 6 of the $104 \mathrm{O}$ and early B stars classified by Walborn \& Blades (1997), that is $\sim 6 \%$, belonged to the $\mathrm{Vz}$ category. If we assume that all 20 bright stars we detected towards N81 (Paper I) are of O or B type, then the lower limit for the fraction of Vz stars in N81 is nearly 35\%, which is quite considerable!

The exact nature and evolutionary stage of $\mathrm{Vz}$ stars is still unknown. Presumably these objects are close to a transition from their formation locus in the HR diagram to the main sequence. However, several issues regarding their properties, which are relevant to our observations of compact low-metallicity $\mathrm{H}$ II regions, remain open:

1. Why do these stars show such weak stellar winds? Are their mass loss rates compatible with expectations from "normal" O stars, i.e. due to their reduced luminosity compared to stars with similar effective temperatures and representative for massive stars in their earliest evolutionary stage?

2. Are these objects truly on the ZAMS, blueward or redward of it? The presence or absence of massive stars on the ZAMS - corresponding to the locus of completely homogeneous objects initiating $\mathrm{H}$-burning - yields information on the star formation process. Indeed, the apparent lack of Galactic OB stars close the ZAMS could be due to the hiding of such stars in their parental cocoon (Garmany et al. 1982) or explained by the progressive redward bending of the upper part of the birthline due to moderate mass accretion rates in an accretion scenario for these stars (Bernasconi \& Maeder 1996). In the former case the position of the "earliest" star visible thus provides constraints on the duration of the hidden phase. For the latter scenario, the position of the bluest stars constrains the accretion rate $\dot{M}_{\text {acc }}$; the existence of massive stars close to the ZAMS requires high values of $\dot{M}_{\text {acc }}$ (Norberg \& Maeder 2000; Behrend \& Maeder 2001).

Given the present uncertainties on the $T_{\text {eff }}$ determination of the N81 stars, one may speculate that some of the objects are indeed hotter than the ZAMS, as indicated by Fig. 3. If true, this "blue straggler" like behavior could be indicative of stellar collisions (e.g. Benz \& Hills 1987), and thus a signature of formation of massive stars via this process, as advocated among others by Bonnell et al. (1998).

3. Could some of the N81 stars still be accreting pre-main sequence objects? The stars with smaller $M_{V}$ magnitudes (\#3, 4, 8, 11, and 13) have estimated luminosities slightly above the probable high luminosity Herbig Ae/Be pre-main sequence stars found by Lamers et al. (1999) and de Wit et al. (2001) in the LMC and SMC. Their location in the HRD also coincides with the region where the predicted birthlines follow quite closely the ZAMS. Given the strong indications for a very young age of N81, it is thus conceivable that our objects are still accreting mass as part of their formation process. The redshifted C IV profiles in the above cited objects could be an indication of ongoing accretion.

4. Are Vz stars related to low metallicity? Vz stars have also been detected in the Milky Way by Walborn and co-workers (private communication). Therefore, it appears unlikely that they are due to a simple metallicity effect.

While at present these answers remain fairly speculative, our upcoming determinations of stellar and wind parameters and further studies of similar objects should hopefully shed more light onto these issues related to the formation and early evolution of massive stars.

\section{Conclusions}

Our HST/STIS observations of the brightest massive stars powering the high excitation compact $\mathrm{H}$ II region SMC N81 reveal that the stars have strikingly weak wind profiles and a pronounced sub-luminosity which are clear indications of their early evolutionary state. Most likely they belong to the $\mathrm{Vz}$ category of massive Magellanic 
Cloud stars (Walborn \& Parker 1992; Walborn \& Blades 1997), which are very young stars just arriving at the ZAMS or already located near it. These stars may also serve as templates for newborn massive stars of distant metal-poor galaxies which cannot be individually resolved. Therefore, a more detailed study and modeling of their properties is highly desirable as it will help shed some more light on the intricacies and consequences of the early stages in stellar evolution.

Acknowledgements. We would like to thank the referee for a critical reading of the paper which contributed to its improvement. We are also grateful to Dr. Nolan R. Walborn (Space Telescope Science Institute) for very helpful comments and suggestions. VC would like to acknowledge the financial support for this work provided by NASA through grant number GO-8246 from the STScI, which is operated by the Association of Universities for Research in Astronomy, Inc., under NASA contract 26555. DS, FM, and MH-M received partial support from the French "Programme National de Physique Stellaire" (PNPS).

\section{References}

Beech, M., \& Mitalas, R. 1994, ApJS, 95, 517

Behrend, R., \& Maeder, A. 2001, A\&A, 373, 122

Benz, W., \& Hills, J. G. 1987, ApJ, 323, 614

Bernasconi, P. A., \& Maeder, A. 1996, A\&A, 307, 829

Bonnell, I. A., Bate, M. R., \& Zinnecker, H. 1998, MNRAS, 298, 93

Churchwell, E. 1990, A\&AR, 2, 79

Davies, R. D., Elliott, K. H., \& Meaburn, J. 1976, MNRAS, 81,89

de Wit, W. J., Beaulieu, J. P., Lamers, H. J. G. L. M., Lesquoy, E., \& Marquette, J.-B. 2001, A\&A, submitted

Di Benedetto, G. P. 1997, ApJ, 486, 60

Garmany, C., Conti, P. S., \& Chiosi, C. 1982, ApJ, 263, 777

Garmany, C., Conti, P. S., \& Chiosi, C. 1985, ApJ, 293, 407

Hanson, M. M., Conti, P. S., \& Rieke, M. J. 1996, ApJS, 107, 281

Henize, K. G. 1956, ApJS, 2, 315

Heydari-Malayeri, M., Le Bertre, T., \& Magain, P. 1988, A\&A, 195,230

Heydari-Malayeri, M., Rosa, M., Zinnecker, H., Deharveng, L., \& Charmandaris, V. 1999, A\&A, 344, 848 (Paper I)

Heydari-Malayeri, M., Charmandaris, V., Deharveng, L., Rosa, M. R., \& Zinnecker, H. 1999b, A\&A, 347, 841
Heydari-Malayeri, M., Rosa, M. R., Charmandaris, V., Deharveng, L., \& Zinnecker, H. 1999c, A\&A, 352, 665

Heydari-Malayeri, M., Charmandaris, V., Deharveng, L., et al. 2001a, A\&A, 372, 495

Heydari-Malayeri, M., Charmandaris, V., Deharveng, L., et al. 2001b, A\&A, 372, 527

Hutchings, J. B. 1982, ApJ, 255, 70

Israel, F. P., \& Koornneef, J. 1988, A\&A, 190, 21

Israel, F. P., \& Koornneef, J. 1991, A\&A, 248, 404

Israel, F. P., Johansson, L. E. B., Lequeux, J., et al. 1993, A\&A, 276, 25

Kaltcheva, N. T., \& Georgiev, L. N. 1992, MNRAS, 259, 166

Lamers, H. J. G. L. M., Beaulieu, J. P., \& de Wit, W. J. 1999, A\&A, 341, 827

Lejeune, T., \& Schaerer, D. 2001, A\&AS, 366, 538

Martins, F., et al. 2001, in preparation

Norberg, P., \& Maeder, A. 2000, A\&A, 359, 1025

Palla, F., \& Stahler, S. W. 1990, ApJ, L47

Prinja, R. K., \& Crowther, P. A. 1998, MNRAS, 300, 828

Relyea, L. J., \& Kurucz, R. L. 1978, ApJS, 37, 45

Shu, F. R., Adams, F. C., \& Lizano, S. 1987, ARA\&A, 25, 23

Smith Neubig, M. M., \& Bruhweiler, F. C. 1997, AJ, 114, 1951

Smith Neubig, M. M., \& Bruhweiler, F. C. 1999, AJ, 117, 2856

Vacca W. D., Garmany C. D., \& Shull, J. M. 1996, ApJ, 460, 914

Walborn, N. R. 1972, AJ, 77, 312

Walborn N. R., \& Blades, J. C. 1997, ApJS, 112, 457

Walborn, N. R., \& Fitzpatrick, E. L. 1990, PASP, 102, 379

Walborn, N. R., \& Panek, R. J. 1985, ApJ, 291, 806

Walborn, N. R., \& Parker, J. Wm. 1992, ApJ, 399, L87

Walborn, N. R., Nichols-Rohlin, J., \& Panek, R. J. 1985, International Ultraviolet Explorer Atlas of O-Type Spectra from 1200 to $1900 \AA$ (NASA RP-1155)

Walborn, N. R., Parker, J. Wm., \& Nichols, J. S. 1995a, International Ultraviolet Explorer Atlas of B-Type Spectra from 1200 to $1900 \AA$ (NASA RP-1363)

Walborn, N. R., Lennon, J. L., Haser, S. M., et al. 1995b, PASP, 107, 104

Walborn, N. R., Lennon, J. L., Heap, S. R., et al. 2000, PASP, 112,1243

Werner, K., \& Rauch, T. 2001, in Eta Carinae and other Mysterious Stars, ASP Conf. Ser., ed. T. Gull, et al. [astro-ph/0102006]

Willis, A. J., van der Hucht, K. A., Conti, P. S., \& Garmany, D. 1986, A\&AS, 63, 417

Woodgate, B. E. 1998, PASP, 110, 1183

Yorke, H. W., \& Krügel, E. 1977, A\&A, 54, 183 